\title{
Erratum to: Effects of Renal Sympathetic Denervation on Arterial Stiffness and Blood Pressure Control in Resistant Hypertensive Patients: A Single Centre Prospective Study
}

\author{
Matteo Baroni $^{1,2} \cdot$ Stefano Nava $^{1,2} \cdot$ Luca Giupponi $^{1,2} \cdot$ Paolo Meani $^{1,2}$. \\ Francesco Panzeri ${ }^{1,2} \cdot$ Marisa Varrenti ${ }^{1,2}$ - Alessandro Maloberti ${ }^{1,2}$. \\ Francesco Soriano $^{3}$ - Antonio Maria Agrati $^{4}$ - Giovanni Ferraro ${ }^{4} \cdot$ Fabrizio Colombo ${ }^{4}$. \\ Antonio Rampoldi ${ }^{5}$ Giuseppe Mancia $^{2}$ - Paola Colombo ${ }^{3}$ - Silvio Klugmann ${ }^{3}$. \\ Cristina Giannattasio ${ }^{1,2}$
}

Published online: 20 November 2015

(C) Springer International Publishing Switzerland 2015

\section{Erratum to: High Blood Press Cardiovasc Prev DOI 10.1007/s40292-015-0121-4}

Unfortunately, first and last name of all authors have been interchanged in the original publication. The author names are corrected here:

Matteo Baroni ${ }^{1,2}$, Stefano Nava ${ }^{1,2}$, Luca Giupponi ${ }^{1,2}$, Paolo Meani $^{1,2}$, Francesco Panzeri ${ }^{1,2}$, Marisa Varrenti ${ }^{1,2}$, Alessandro Maloberti ${ }^{1,2}$, Francesco Soriano $^{3}$, Antonio Maria Agrati ${ }^{4}$, Giovanni Ferraro ${ }^{4}$, Fabrizio Colombo ${ }^{4}$, Antonio Rampoldi ${ }^{5}$, Giuseppe Mancia ${ }^{2}$, Paola Colombo ${ }^{3}$, Silvio Klugmann ${ }^{3}$, Cristina Giannattasio ${ }^{1,2}$

The original article has been updated accordingly.

The online version of the original article can be found under doi:10.1007/s40292-015-0121-4.

Matteo Baroni

bimatteo@gmail.com

1 Cardiology IV, Cardiovascular "A' De Gasperis"

Department, Niguarda Ca' Granda Hospital, Piazza Ospedale

Maggiore 3, 20161 Milan, Italy

2 Health Science Department, Milan-Bicocca University, Milan, Italy

3 Cardiology I, Cardiovascular “A' De Gasperis” Department, Niguarda Ca' Granda Hospital, Milan, Italy

4 Internal Medicine Department, Niguarda Ca' Granda Hospital, Milan, Italy

5 Interventional Radiology, Niguarda Ca' Granda Hospital, Milan, Italy 